\title{
Platinum Isotope Variations in Chondrite Meteorites
}

\author{
A.C. HUNT*, L. ISELI AND M. SCHÖNBÄCHLER
}

ETH Zürich, Department of Earth Sciences, Switzerland

(*correspondence: alison.hunt@erdw.ethz.ch)

Nucleosynthetic $s$-process variations in bulk chondrites were determined for elements including $\mathrm{Zr}$, Mo and $\mathrm{Ru}$ [e.g., 1-3]. Conversely, no variations have been detected in chondrites for heavier elements such as Hf and Os [4,5]. High-precision $\mathrm{Pt}$ isotope studies of iron meteorites also reveal no $s$-process variations [6,7]. This disconnect suggests high metallicity asymptotic giant branch stars are the dominant contributors of presolar grains to the Solar System [8]. Additionally, Pt isotope ratios are altered by exposure to galactic cosmic rays (GCR) [9], and have been exploited as a neutron dosimeter, e.g., for $\mathrm{W}$ and $\mathrm{Pd}[8,10]$. Here, we present $\mathrm{Pt}$ isotope ratios in a range of chondrites to assess both nucleosynthetic and GCR variations.

Chondrites including Monze (L6), St. Severin (LL6), Parnallee (LL3.6), Richardton (H5), Indarch (EH) and Daniel's Kuil (EL) were digested using a fire assay technique and processed though ion exchange chemistry [11]. Platinum fractions were measured using a Neptune Plus MC-ICP-MS. This yields external reproducibilities of 0.75 for $\varepsilon^{192} \mathrm{Pt}$ and 0.09 for $\varepsilon^{196} \mathrm{Pt}$ (2SD) [11].

The selected chondrites have exposure ages of $\sim 5-30$ Myr [12,13]. Using the model of [9], this is predicted to result in GCR variations to $\varepsilon^{196} \mathrm{Pt}$ that are within analytical uncertainty. However, [7] found that while these models successfully predict the trend between $\varepsilon^{192} \mathrm{Pt}$ and $\varepsilon^{196} \mathrm{Pt}$ for iron meteorites, they do not fully account for the magnitude of measured variations. New Pt isotope data suggest this is also the case for chondrites. This has implications for all isotope systems that are affected by GCR. Using the relative susceptibilities to GCR of $\varepsilon^{196} \mathrm{Pt}$ and $\varepsilon^{182} \mathrm{~W}$ [10] measurable offsets to $\varepsilon^{182} \mathrm{~W}$ are predicted, casting doubt on precise ages determined for chondrites using the Hf-W chronometer.

References: [1] Akram et al. (2015) GCA 165, 484-500. [2] Burkhardt et al. (2011) EPSL 312, 390-400. [3] FischerGödde et al. (2017) Nature 541, 525-527. [4] Sprung et al. (2010) EPSL 295, 1-11. [5] Walker (2012) EPSL 351-352, 36-44. [6] Peters et al. (2015) Chem. Geol. 413, 132-145. [7] Hunt et al. (2017) GCA 216, 82-95. [8] Ek et al. (2020) Nat. Astron. [9] Leya \& Masarik (2013) MAPS 48, 665-685. [10] Kruijer et al. (2013) EPSL 361, 162-172. [11] Hunt et al. (2017) GGR 41, 633-647. [12] Crabb and Anders (1981) GCA 45, 2443-2464. [13] Zähringer (1962) Z. Naturforsch. A $17,460-471$. 